\title{
A Plasmonic Dimple Lens for Nanoscale Focusing of Light
}

\author{
Shantha Vedantam, ${ }^{*, \dagger}$ Hyojune Lee, ${ }^{\dagger}$ Japeck Tang, ${ }^{\dagger}{ }^{\text {Josh Conway }}{ }^{\dagger}$ \\ Matteo Staffaroni, ${ }^{\dagger}$ and Eli Yablonovitch ${ }^{\dagger, \neq}$
}

\author{
Electrical Engineering Department, University of California, Los Angeles, California \\ 90095, and Department of Electrical Engineering \& Computer Sciences, University of \\ California, Berkeley, California 94720
}

Received May 24, 2009; Revised Manuscript Received August 10, 2009

\begin{abstract}
Focusing electromagnetic energy to subwavelength dimensions has become an increasingly active field of research for a variety of applications such as heat-assisted magnetic recording, nanolithography, and nanoscale optical characterization of biological cells and single molecules using the near-field scanning optical microscopy technique. Double-sided surface plasmons in a metal-insulator-metal (MIM) geometry can have very small wavelengths for dielectric of thickness of less than $10 \mathrm{~nm}$. A tapered dielectric structure sandwiched between metal can be used to efficiently couple electromagnetic energy from free space photons to the plasmonic wavelengths at the nanoscale. In this paper, we present the fabrication and characterization of a novel MIM plasmonic lens structure.
\end{abstract}

Conventional optics can only focus light to a diffractionlimited spot size of approximately $\lambda / 2 n$ in a medium with refractive index $n$. For visible wavelengths, the diffractionlimited spot is on the order of $100 \mathrm{~nm}$ even in a high index transparent material like diamond $(n \sim 2.4)$. In order to further focus light to smaller dimensions, one approach has been to use the confinement of electric fields that occurs around a sharp tip. ${ }^{1,2}$ Near-field scanning optical microscopy (NSOM) is another standard technique for confining light to minimum spot size of $\sim 100 \mathrm{~nm}$ using metallized pulled optical fibers with a nanoscale aperture. The transmission through these tapers is evanescent, and further reduction of the aperture size results in significant reduction in throughput. ${ }^{3}$ Throughput is not necessarily the right figure-of-merit in any case, since the effect of the focusing structure is to concentrate electromagnetic energy within a small electric or magnetic dipole volume, and it is this transfer efficiency of energy to the dipole structure that is important, and not necessarily the throughput. Bow-tie antennas and C-shaped aperture antennas ${ }^{4-6}$ are alternate optical frequency designs.

Another good approach to confine electromagnetic energy has been to take advantage of the short optical wavelengths of waves running along a metal-dielectric interface, known as surface plasmons. ${ }^{7}$ Grating couplers, similar to antennas, can be used to couple free space light into surface plasmons. Furthermore, a circular grating can be used to focus these

\footnotetext{
* Corresponding author, shantha@ee.ucla.edu.

${ }^{\dagger}$ Electrical Engineering Department, University of California, Los Angeles.

¥ Department of Electrical Engineering \& Computer Sciences, University of California, Berkeley.
}

surface plasmons toward a spot at the center of the circle. This is analogous to a lens for free space light, which results in a higher intensity at the focal point. ${ }^{8}$

Unfortunately, single-sided plasmons have a small spot size only near the surface plasmon frequency of the metal and the dielectric. In contrast, the wavelength of doublesided plasmons can be tuned geometrically by varying the thickness of the gap. It has been shown that the plasmon wavelength of the microstrip transmission line mode in a metal-insulator-metal structure decreases with the thickness of the dielectric. ${ }^{9}$ Thus, one can control the focal spot size by employing double-sided plasmons in a transmission-line structure with an adjustable thickness dielectric. However, this introduces the additional complication of requiring a way to efficiently couple energy from a large dielectric thickness to the smaller thickness, such as a tapered structure. ${ }^{9,10}$ The basic design parameters of a 3D tapered metal-insulatormetal structure for focusing double-sided plasmons to nanometer scale dimensions is discussed in ref 9. The present paper outlines a novel approach to the top-down fabrication of this three-dimensional transmission line nanostructure along with the results of preliminary experimental characterization. ${ }^{11}$

Principle and Design. It has been shown ${ }^{9,12}$ that doublesided surface plasmons can confine light to the nanometer scale by decreasing the dielectric thickness of the metalinsulator-metal stack. Figure 1 shows the dispersion relation of $\mathrm{Au}-\mathrm{SiO}_{2}-\mathrm{Au}$ stack for various dielectric thicknesses using the optical constants from ref 13. It is evident from the graph that for a fixed wavelength of operation, say 633 


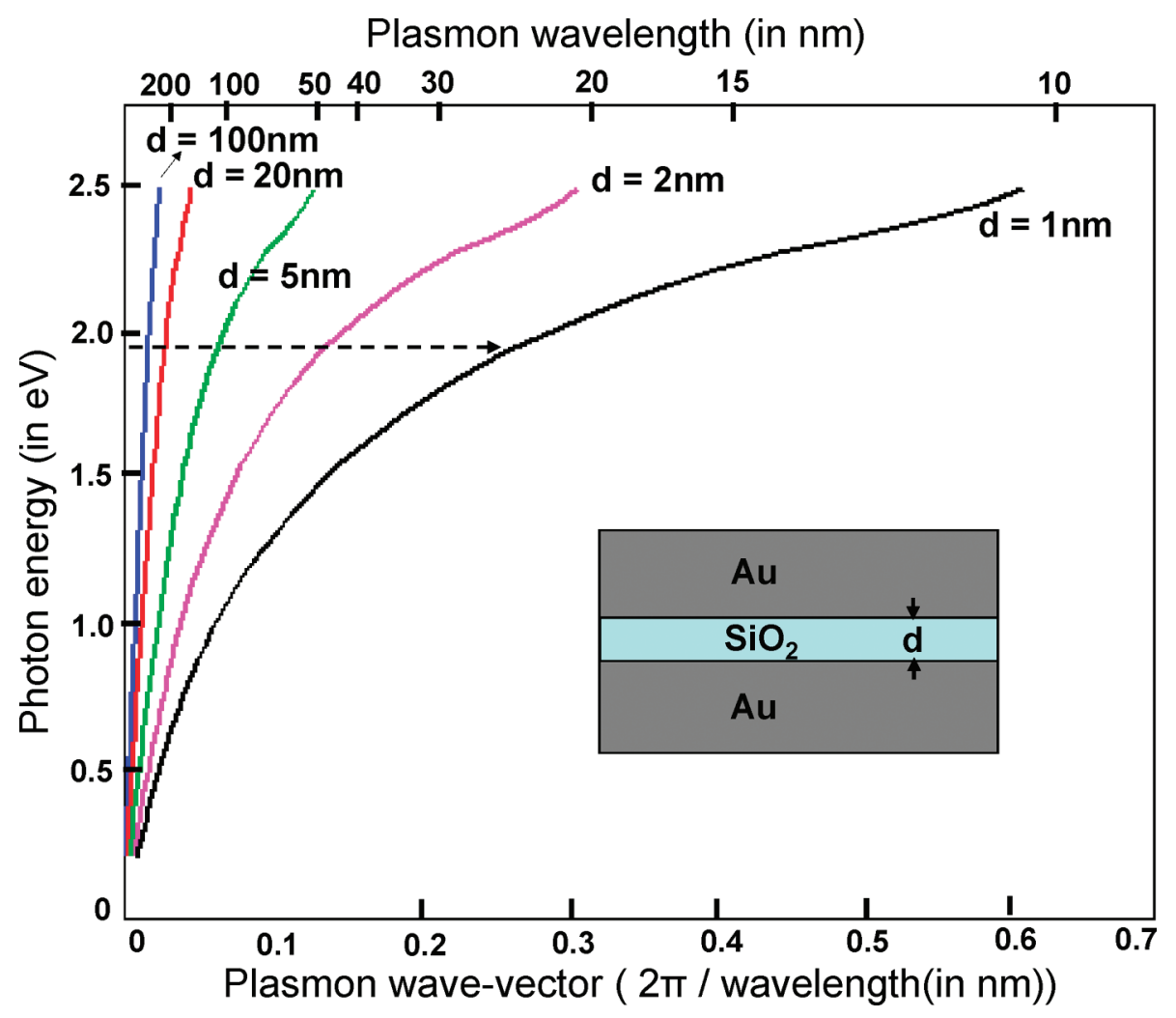

Figure 1. Calculated dispersion relations for the $\mathrm{Au}-\mathrm{SiO}_{2}-\mathrm{Au}$ stack for various thicknesses of the $\mathrm{SiO}_{2}$ layer.

$\mathrm{nm}(1.96 \mathrm{eV})$, the double-sided surface plasmon wavelength can be varied from $330 \mathrm{~nm}$ to less than $30 \mathrm{~nm}$ by varying the thickness of $\mathrm{SiO}_{2}$ from $100 \mathrm{~nm}$ down to $1 \mathrm{~nm}$.

In this work, we employ a tapered MIM structure, as shown in Figure 2a, to focus double-sided surface plasmons by tapering the microstrip transmission line to a sharp point. An optimal taper angle ensures efficient coupling of electromagnetic energy from larger plasmonic wavelengths to nanoscale plasmonic wavelengths with minimum resistive and reflective energy losses. ${ }^{9}$ A circular grating coupler is designed to couple free space visible light into single-sided surface plasmons before they traverse the taper to an outcoupling facet. As the plasmons propagate across the taper, their phase velocity is reduced in proportion to their wavelength. The two-dimensional analogue of the classical $3 \mathrm{D}$ immersion lens, formed in a $2 \mathrm{D}$ transmission line is depicted in Figure 2b. We refer to this focusing structure as a plasmonic dimple lens. We note that this structure is fundamentally different from a classical immersion lens in that the effective index is changing continuously; and that this change can be larger than an order of magnitude. These traits lead to unique properties owing to the extremely high achievable refractive index for MIM plasmonic waveguide mode. The short plasmonic wavelengths at the end of the taper give rise to strong excitation of the optical electric field at the focal tip. Thus, optical focusing is achieved in all three dimensions, confining energy to the nanoscopic tip. The design parameters and field enhancement estimates can be found in Chapter 3 of ref 9.

While the terminal thickness of the dielectric taper and the semicircular shape of the dimple lens determine the smallest achievable 2D spot size of energy focusing, the optimal taper angle determines the efficiency of energy coupling from large plasmonic wavelengths to small plasmonic wavelengths. The most appropriate figure-of-merit which accounts for both of the above can be defined ${ }^{9}$ by $F$ $=c \varepsilon E_{\max }^{2} / P_{\text {in }}$, where $F$ has the dimension of $1 /$ area and it represents the energy focusing area of a lens. It not only represents the $1 /$ spot size of focused energy but also accounts for energy compression along the direction of propagation and energy losses down the taper. This figure-of-merit should be used to compare the different candidate structures such as those mentioned in the introduction. A crude figure-ofmerit of a plasmonic lens can just be simplified to the spot size of the focusing achieved at the focal point of the lens since this can be monitored more easily than the local electric field at the nanoscale.

Fabrication and Measurement. The critical dimension determining the spot size of the focused plasmonic energy structure shown in Figure 2 is the final thickness of the dielectric at the end of the taper. The fabrication of a threedimensional semicircular dimple taper in a dielectric terminating with a thickness of a few nanometers poses a significant challenge. For small dielectric thickness in a MIM structure, double-sided surface plasmons have short wavelengths (see Figure 1), which have large resistive energy losses and hence short propagation lengths. ${ }^{9}$ Since the plasmonic currents are flowing on the metal surfaces, they suffer additional energy loss by scattering via interface roughness. It has been observed that e-beam evaporated gold and silver surfaces typically have surface roughness greater than $2-3 \mathrm{~nm}$ rms due to the formation of grains. ${ }^{14,15}$ To keep 
(a)

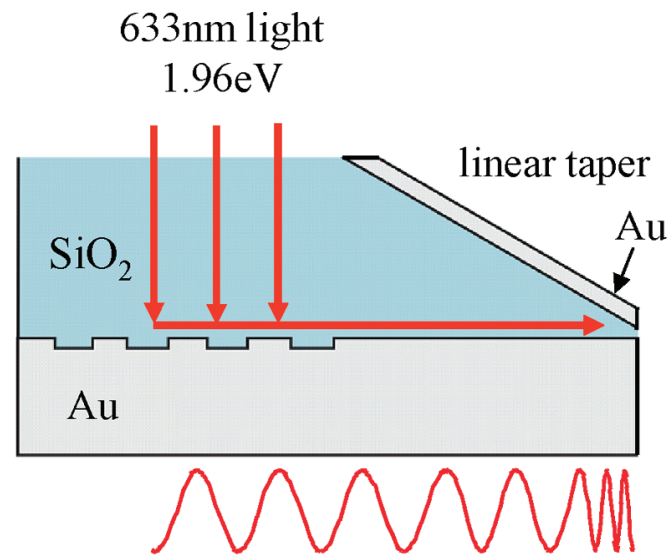

(b)

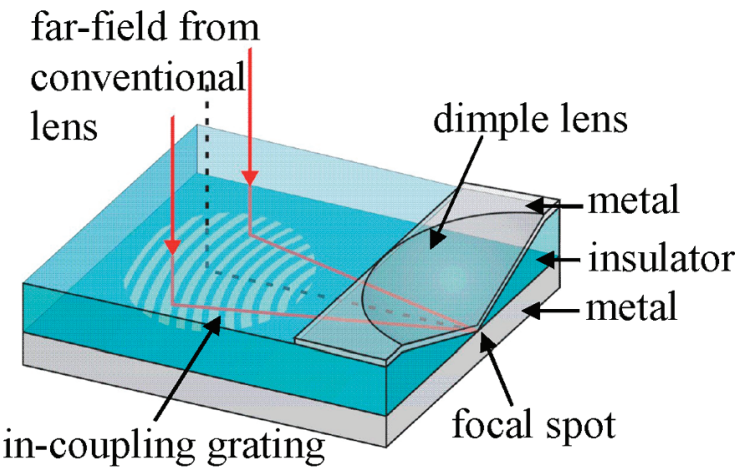

(c)
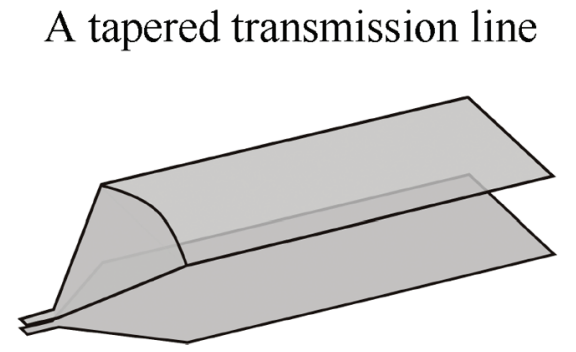

Figure 2. (a) Cross-sectional view schematic of the plasmonic dimple lens. The thickness of $\mathrm{SiO}_{2}$ layer tapers in a vertical direction. The wavelength of double-sided surface plasmons changes along the taper. (b) A perspective view of the plasmonic dimple lens. The focusing action is analogous to that of a tapered transmission line in (c).

this scattering loss low, it is important to ensure smooth surfaces at the two metal-dielectric interfaces that support plasmons. In view of these considerations, we fabricated the plasmonic dimple lens as a $\mathrm{Au}$-dielectric-Au structure where the dielectric consisted of a layer of poly(methyl methacrylate) (PMMA) and a layer of thin silicon nitride. The maturity in silicon processing yields high-quality amorphous oxide and nitride films on a silicon surface a few nanometers in thickness. ${ }^{16,17}$ Also much progress has been made in tailoring smooth grayscale profiles in e-beam and photosensitive resists. ${ }^{18-22}$

Our approach is to fabricate the taper profile in PMMA (a positive e-beam resist) on top of a thin layer of silicon nitride that determines the minimum dielectric thickness of the plasmonic dimple lens. The refractive index of the PMMA is $1.49-1.52$ and is close to that of $\mathrm{SiO}_{2}$. Our process sequence also ensures that gold is deposited onto smooth amorphous dielectric surfaces thereby minimizing the scattering energy loss owing to interface roughness.

The exact process sequence as depicted in Figure 3 emerged from numerous trials involving different materials like metals, dielectrics, polymers, and epoxies, and different process steps like etch and polishing. ${ }^{23}$ Thin silicon nitride of $\sim 10 \mathrm{~nm}$ thickness was deposited on a standard silicon wafer, using a low-pressure chemical vapor deposition (LPCVD) process. Gold islands were then patterned on this smooth silicon nitride surface using photolithography, e-beam evaporation, and liftoff process (Figure 3a). These islands constitute the lower Au layer in the $\mathrm{Au}$-dielectric-Au stack of Figure 2a. They also include the fiducial alignment markers for subsequent e-beam lithography steps and the end point detection markers for the final edge-polishing step.

About $300 \mathrm{~nm}$ of silicon nitride was deposited on top of these islands by a plasma-enhanced chemical vapor deposition (PECVD) process to reduce stress on the thin LPCVD silicon nitride during the subsequent bulk silicon removal step. The patterned side of the sample was bonded to a glass piece using low stress silica-filled epoxy for support during the silicon substrate removal. This stack is shown in Figure 3b. The silicon substrate was thinned down to less than 50 $\mu \mathrm{m}$ using deep reactive ion etching (DRIE). The remaining silicon was removed by wet etching in a 1:1 mixture of HF and $\mathrm{HNO}_{3}$ as depicted in Figure 3c. This mixture etches silicon but etches LPCVD silicon nitride over $10^{3}$ times more slowly.

A semicircular Au grating of $30 \mathrm{~nm}$ height and $390 \mathrm{~nm}$ period was patterned using e-beam lithography, e-beam evaporation, and a resist liftoff process (Figure 3d). A circular dimple profile was then formed in a $100 \mathrm{~nm}$ layer of PMMA with a single spot exposure using e-beam lithography (Figure $3 \mathrm{e})$. Both the grating and the dimple were inspected using a scanning electron microscope (SEM) and an atomic force microscope (AFM), respectively, as can be seen in Figure 4. To form the top $\mathrm{Au}$ layer of $\mathrm{Au}$-dielectric-Au stack as shown in Figure 2a, $100 \mathrm{~nm}$ layer of Au was evaporated on top of the PMMA layer using e-beam evaporation (Figure 3f). This Au layer was then etched to gain optical access to the grating using Ar-ion sputter etch with e-beam patterned SU-8 (a negative e-beam resist) as a mask. Figure $3 \mathrm{~g}$ depicts the stack at this stage. The structure is then bonded to another piece of glass using optically transparent epoxy for additional support during the mechanical polishing step as seen in Figure $3 \mathrm{~h}$. Finally, the sample is polished from the edge until the center of the dimple is exposed at the polished edge facet (Figure 3i). The topographic and phase-shift images from the AFM scan of the polished edge facet are shown in Figure 5.

The fabricated device was characterized using a modified Veeco Aurora-3 NSOM system, which uses shear-force feedback for scanning. A schematic of the measurement setup is shown in Figure 6. The sample was mounted vertically on the NSOM stage so that the polished out-coupling edge of the device points upward. A $633 \mathrm{~nm}$ light from a HeNe laser was focused from the side onto the grating region of the device using a microscope objective $(50 \times, 0.45 \mathrm{NA})$. The 

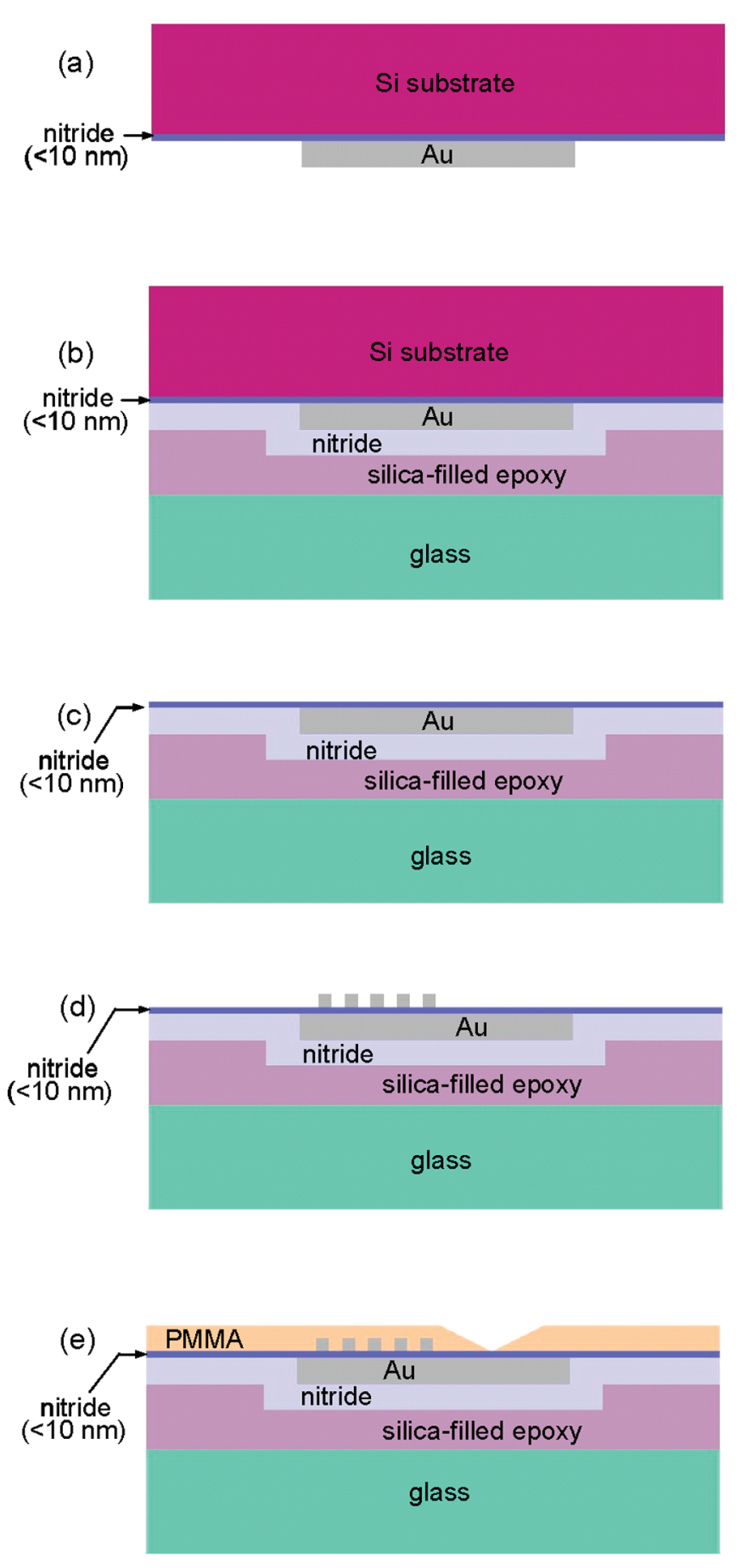

Figure 3. (a) Au islands patterned on $10 \mathrm{~nm}$ LPCVD SiN on a Si-substrate. (b)These islands are coated with $300 \mathrm{~nm}$ of PECVD SiN and bonded to a glass using silica-filled epoxy. (c) Si-substrate is completely etched away with $\mathrm{HNO}_{3}+\mathrm{HF}$ after thinning it down with the DRIE process. (d) Semicircular grating coupler is fabricated using e-beam lithography, followed by Au metal e-beam evaporation and resist lift-off. (e) The dimple profile in PMMA is patterned with e-beam lithography. (f) The second layer of Au is deposited by e-beam evaporation. (g) With patterned SU-8 resist as a mask, this Au layer over the grating coupler is etched away with Ar-sputter etch. (h) The sample is bonded to glass using optically transparent epoxy. (i) This stack is mechanically polished from one edge until the point when the circular dimple profile in PMMA is polished halfway through. out-coupled light from the device was collected with a photomultiplier tube (PMT) through a commercially available pulled-fiber NSOM probe.

The sample was scanned with the NSOM probe to acquire both the topographic image and the scanned optical image at the same time. Panels a and b of Figure 7 show good correlation between the region of high intensity of light
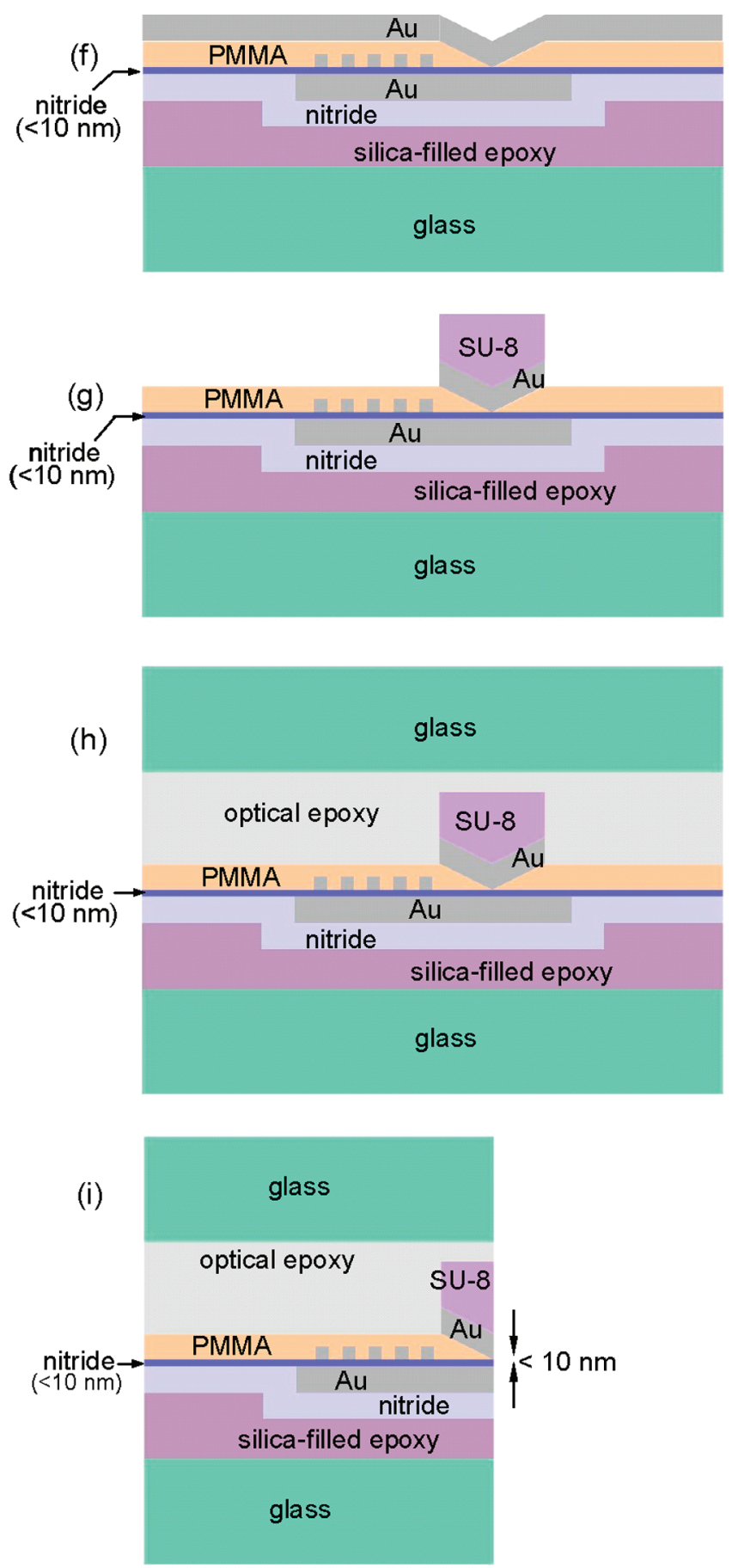

in the optical image with the dimple region of the device in the topographic scan. Furthermore the spot size formed by the dimple lens is smaller than that formed by the circular grating coupler alone, as evident from panels $b$ and $d$ of Figure 7, respectively. It is important to note that the absolute spot size measurement in panels $b$ and $d$ of Figure 7 is believed to be limited by the size of the pulled-fiber probe 


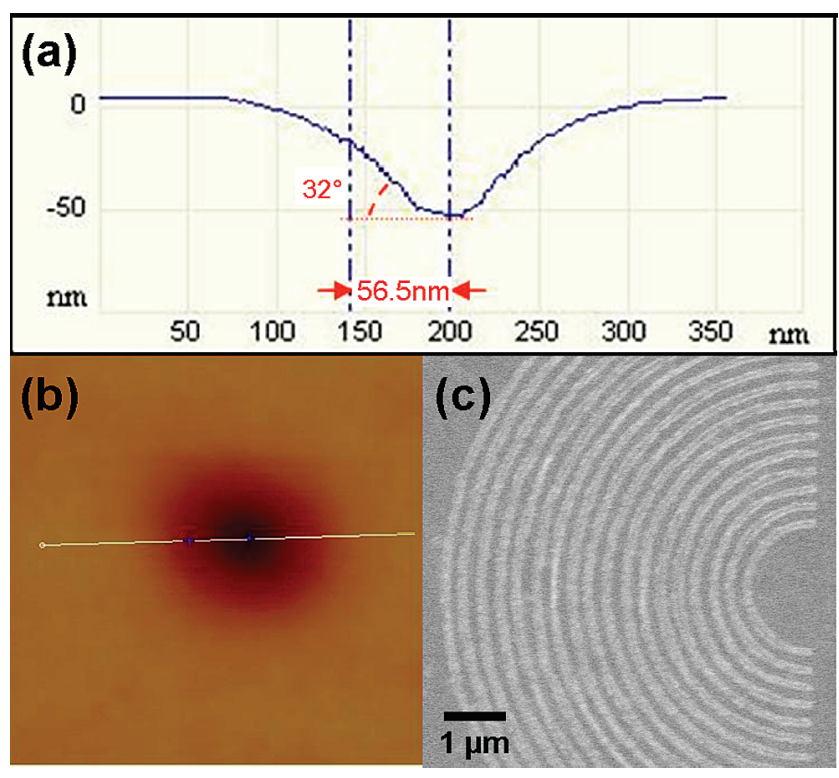

Figure 4. (a) Topographic cross sectional scan of dimple and (b) 2D top surface image acquired from the topographic AFM scan of the dimple profile in PMMA. The size of the scan is $400 \mathrm{~nm} \times$ $400 \mathrm{~nm}$. (c) SEM image of the circular grating coupler.

aperture (which varies between 100 and $150 \mathrm{~nm}$ ) used in NSOM measurement system.

Conclusions. We have designed and fabricated a novel plasmonic dimple lens structure in $\mathrm{Au}$-dielectric-Au geometry for focusing visible light to the nanoscale. Comparison of measurements of the circular grating coupler alone and the circular grating coupler together with a dimple lens reveal additional focusing provided by the plasmonic dimple lens. However, the measurement of the final spot size produced by the dimple lens was not possible due to the resolution limit of the pulled-fiber NSOM probe used in the experiment. Our plasmonic lens is capable of focusing light to spot sizes smaller than the aperture size of the commercially available pulled-fiber NSOM probes.

The fabrication strategy is such that the critical dimension of this plasmonic lens is controlled by the layer thickness of the dimple gap and NOT by the transverse lithographic limitations of electron beam or ion beam processes or equipment. The mechanical edge-polishing step in our fabrication sequence lends itself to be easily adopted by the process flow of the magnetic read-write head of a commercial hard drive. Thus, this structure could potentially be an excellent candidate for the heat-assisted magnetic recording (HAMR) technology, where the plasmonic dimple lens would be used to focus light to provide the strong local heating required for the next generation higher density hard drives. ${ }^{24,25}$

There are possible improvements in our diagnostics to address the difficulty in measuring the local peak electric field. The limitations of measuring the spot size of the dimple lens using a pulled-fiber aperture probe may be overcome by using a sharp apertureless probe tip to scatter the energy at the focal point and collect this signal in the far-field ${ }^{26}$ while scanning the out-coupling facet of the dimple lens. Another approach is to use a negative-tone photoresist ${ }^{27,28}$ that is
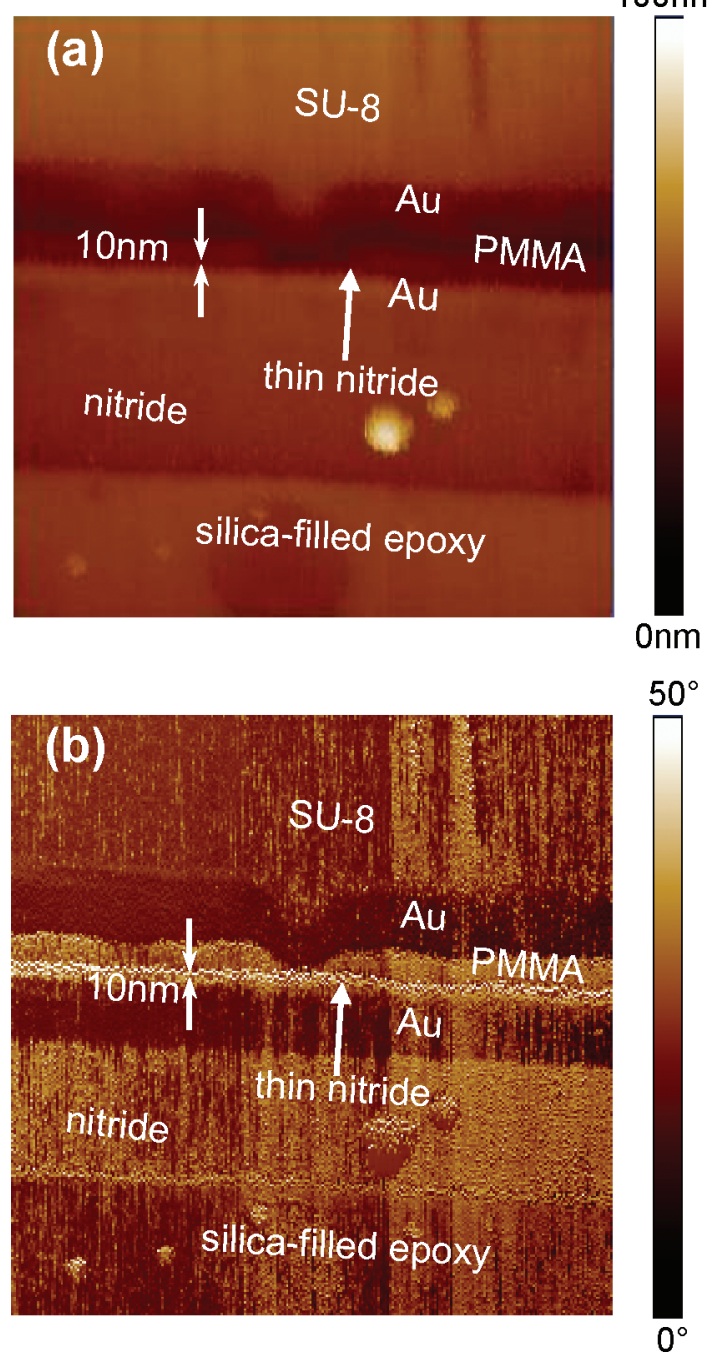

Figure 5. (a) Topographic and (b) out-of-phase tapping mode response of the AFM image of the out-coupling edge after mechanical edge-polishing. The size of the scan is $1.5 \mu \mathrm{m} \times 1.5$ $\mu \mathrm{m}$. A good smooth polish, with minimal polishing relief, makes distinguishing the different layers in the topographic image difficult. The out-of-phase-tapping mode response image helps distinguish the different layers in the image.

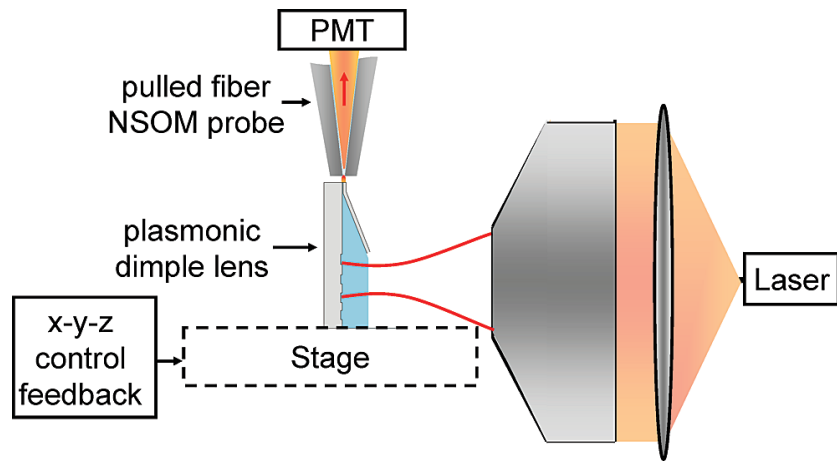

Figure 6. Schematic of the experimental setup to characterize the plasmonic dimple lens using near-field scanning optical microscopy (NSOM).

sensitive to the $633 \mathrm{~nm}$ laser excitation wavelength. Such a photoresist is suitably applied to the out-coupling facet of the dimple lens and gets exposed to the energy at the focal 

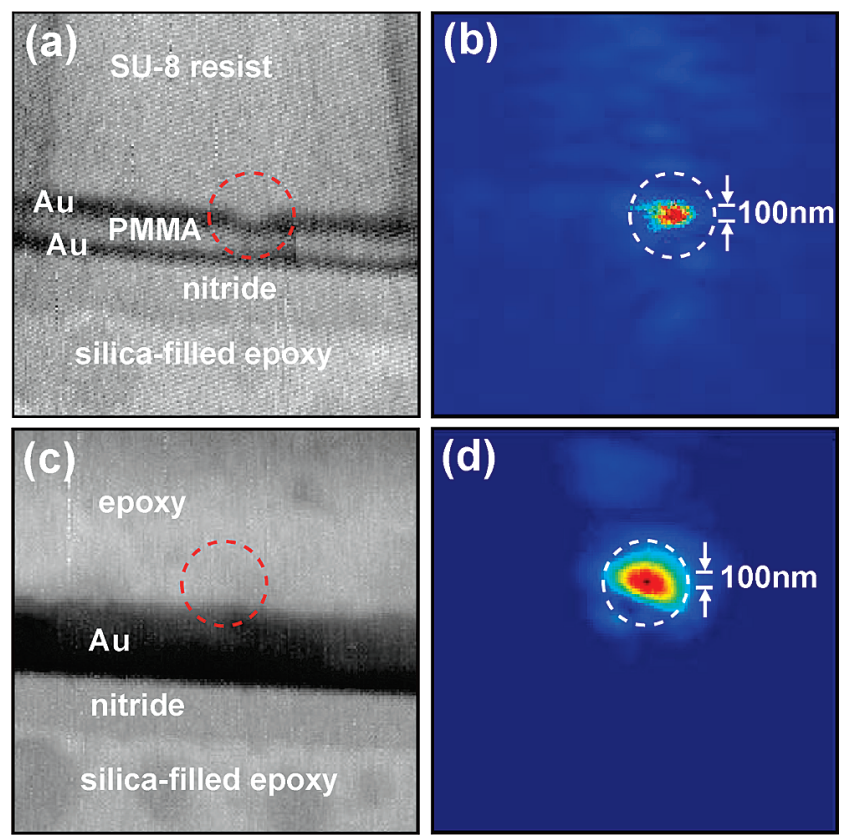

Figure 7. (a) Topographic and (b) scanned optical images of the out-coupling facet of the plasmonic dimple lens obtained by the NSOM technique. (c) Parallel plate waveguides, without a plasmonic dimple lens. Topographic image and (d) corresponding scanned optical images of the polishing facet of the circular grating coupler only (without a plasmonic dimple lens). All the scans are $2.5 \mu \mathrm{m} \times 2.5 \mu \mathrm{m}$.

point when the excitation laser source is turned on. After this, the photoresist can be developed in a developer solution so that the unexposed photoresist is washed away, leaving behind a nanospeck of resist at the focal point. A subsequent AFM scan of the out-coupling facet will determine the spot size of the focused energy. We hope that our dimple lens will be better characterized in the future with at least one of the aforementioned methods.

Acknowledgment. We gratefully acknowledge the support given by the NSF Nanoscale Science and Engineering Center for Scalable and Integrated Nanomanufacturing (SINAM) under the Award Number DMI-0327077 and the Defense Microelectronics Activity (DMEA) under the Agreement Number H94003-06-2-0607, and the Plasmonics MURI No. FA 9550-04-1-0434. We would also like to thank the UCLA Nanoelectronics Research Facility (NRF) where this fabrication process sequence is developed.

\section{References}

(1) Novotny, L.; Pohl, D. W.; Hecht, B. Scanning near-field optical probe with ultrasmall spot size. Opt. Lett. 1995, 20, 970-972.

(2) Chimmalgi, A.; Choi, T. Y.; Grigorapoulos, C. P.; Komvopoulos, K. Femtosecond laser aperturless near-field nanomachining of metals assisted by scanning probe microscopy. Appl. Phys. Lett. 2003, 82, 1146-1148.

(3) Bethe, H. Theory of diffraction by small holes. Phys. Rev. 1944, 66, 163-182.

(4) Sendur, K; Challener, W. Near-field radiation of bow-tie antennas and apertures at optical frequencies. J. Microsc. 2003, 210, 279-283.
(5) Yu, N; Cubukcu, E; Diehl, L; Bour, D; Corzine, S; Zhu, J; Höfler, G; Crozier, K. B.; Capasso, F. Bowtie plasmonic quantum cascade laser antenna. Opt. Express 2007, 15, 13272-13281.

(6) Matteo, J. A.; Fromm, D. P.; Yuen, Y.; Schuck, P. J.; Moerner, W. E.; Hesselink, L. Spectral analysis of strongly enhanced visible light transmission through single C-shaped nanoapertures. Appl. Phys. Lett. 2004, 85, 648-650.

(7) Barnes, W. L.; Dereux, A.; Ebbesen, T. W. Surface plasmon subwavelength optics. Nature 2003, 424, 824-830.

(8) Steele, J. M.; Liu, Z. W.; Wang, Y.; Zhang, X. Resonant and nonresonant generation and focusing of surface plasmons with circular gratings. Opt. Express 2006, 14, 5664-5670.

(9) Conway, J. A. Efficient Optical Coupling to the Nanoscale. Ph.D. thesis, University of California, Los Angeles, 2006, http://optoelectronics.eecs.berkeley.edu/ThesisJosh.pdf.

(10) Pile, D. F. P.; Gramotnev, D. K. Adiabatic and nonadiabatic nanofocusing of plasmons by tapered gap plasmon waveguides. Appl. Phys. Lett. 2006, 89, 041111-1.

(11) Lee, H; Vedantam, S.; Tang, J.; Conway, J.; Staffaroni, M.; Yablonovitch, E. Experimental Demonstration of Optical Nanofocusing by a Plasmonic Dimple Lens. Plasmonics and Metamaterials, OSA Technical Digest; Optical Society of America: Washington, DC, 2008;paper MMA2 (available on CD).

(12) Miyazaki, H. T.; Kurokawa, Y. Squeezing visible light waves into a 3-nm-thick and 55-nm-long plasmon cavity. Phys. Rev. Lett. 2006, 96, 097401-1.

(13) Johnson, P. B.; Christy, R. W. Optical Constants of the Noble Metals. Phys. Rev. B 1972, 6, 4370-4379.

(14) Bendavid, A.; Martin, P. J.; Wieczorek, L. Morphology and optical properties of gold thin films prepared by filtered arc deposition. Thin Solid Films 1999, 354, 169-175.

(15) Petrovic, R.; Strbac, S.; Bundaleski, N.; Rakocevic, Z. Surface roughness minimum: Ag thin layer deposited on glass. J. Serb. Chem. Soc. 2001, 66, 483-490.

(16) Muller, D. A.; Sorsch, T.; Moccio, S.; Baumann, F. H.; EvansLutterodt, K.; Timp, G. The electronic structure at the atomic scale of ultrathin gate oxides. Nature 1999, 399, 758-761.

(17) Shi, X.; Shriver, M.; Zhang, Z.; Higman, T.; Campbell, S. A. Properties of high-k/ultrahigh purity silicon nitride stacks. J. Vac. Sci. Technol., A 2004, 22, 1146-1151.

(18) Stauffer, J. M.; Oppliger, Y.; Regnault, P.; Baraldi, L.; Gale, M. T. Electron beam writing of continuous resist profiles for optical applications. J. Vac. Sci. Technol., B 1992, 10, 2526-2529.

(19) Hu, F.; Lee, S.-Y. Dose control for fabrication of grayscale structures using a single step electron-beam lithographic process. J. Vac. Sci. Technol., B 2003, 21, 2672-2679.

(20) Sung, J. W.; Hockel, H.; Brown, J. D.; Johnson, E. G. Development of a two-dimensional phase-grating mask for fabrication of an analogresist profile. Appl. Opt. 2006, 45, 33-43.

(21) Sure, A.; Dillon, T.; Murakowski, J.; Lin, C.; Pustai, D.; Prather, D. W. Fabrication and characterization of three-dimensional silicon tapers. Opt. Express 2003, 11, 3555-3561.

(22) Vivien, L.; Le Roux, X.; Laval, S.; Cassan, E.; Morini, D. M. Design, Realization, and Characterization of 3-D Taper for Fiber/Micro Waveguide Coupling. IEEE J. Sel. Top. Quantum Electron. 2006, 12, 1354-1358.

(23) Vedantam, S.; Lee, H.; Tang, J.; Conway, J.; Staffaroni, M.; Lu, J.; Yablonovitch, E. Nanoscale fabrication of a plasmonic dimple lens for nano-focusing of light. Proc. SPIE 2007, 6641, 66411J.

(24) Challener, W. A.; Mcdaniel, T. W.; Mihalcea, C. D.; Mountfield, K. R.; Pelhos, K.; Sendur, I. K. Light Delivery Techniques for Heat-Assisted Magnetic Recording. Jpn. J. Appl. Phys. 2003, 42, 981-988.

(25) Mcdaniel, T. W.; Challener, W. A.; Sendur, K. Issues in Heat-Assisted Perpendicular Recording. IEEE Trans. Magn. 2003, 39, 1972-1979.

(26) Yang, T. J.; Lessard, G. A.; Quake, S. R. An apertureless near-field microscope for fluorescence imaging. Appl. Phys. Lett. 2000, 76, 378380 .

(27) Srituravanich, W.; Fang, N.; Sun, C.; Zhang, X. Plasmonic Nanolithography. Nano Lett. 2004, 4, 1085-1088.

(28) Sundaramurthy, A.; Schuck, P. J.; Conley, N. R.; Fromm, D. P.; Kino, G. S.; Moerner, W. E. Toward Nanometer-Scale Optical Photolithography: Utilizing the Near-Field of Bowtie Optical Nanoantennas. Nano Lett. 2006, 6, 355-360.

NL9016368 